\title{
Homogenate Extraction of Crocins from Saffron Optimized by Response Surface Methodology
}

\author{
Yingpeng Tong $\mathbb{D}^{1},{ }^{1}$ Yu Jiang, ${ }^{1}$ Dan Guo, ${ }^{1}$ Yongqiu Yan, ${ }^{1}$ Shiping Jiang, ${ }^{1}$ Yu Lu, \\ S. Zahra Bathaie, ${ }^{2}$ and Ping Wang $\left(^{1}{ }^{1}\right.$ \\ ${ }^{1}$ College of Pharmaceutical Sciences, Zhejiang University of Technology, Hangzhou 310014, China \\ ${ }^{2}$ Department of Clinical Biochemistry, Faculty of Medical Sciences, Tarbiat Modares University, P.O. Box 14115-133, Tehran, Iran
}

Correspondence should be addressed to Ping Wang; wangping45@zjut.edu.cn

Received 29 August 2017; Accepted 12 October 2017; Published 18 January 2018

Academic Editor: Mostafa Khajeh

Copyright (C) 2018 Yingpeng Tong et al. This is an open access article distributed under the Creative Commons Attribution License, which permits unrestricted use, distribution, and reproduction in any medium, provided the original work is properly cited.

Saffron, which has many kinds of biological activities, has been widely used in medicine, cosmetics, food, and other fields of health promotion industries. Crocins are the main component of saffron (Crocus sativus L.). At present, most of the extraction methods for crocins require long time or special instruments to complete the process and some of them are not suitable for industrial production at present. In this article, homogenate extraction technology which is a convenient and efficient method was developed for crocins extraction from saffron. Firstly, the influences of extraction voltage, extraction time, ethanol concentration, and temperature on crocins yield were studied by single factor experiments; and then response surface methodology (RSM) was used to optimize levels of four variables based on the result of single factor experiments. Results showed that the optimum extraction process conditions for crocins were as follows: extraction voltage, $110 \mathrm{~V}$; ethanol concentration, $70 \%$; extraction temperature, $57^{\circ} \mathrm{C}$; and extraction time, $40 \mathrm{~s}$. Based on these conditions, the extraction yield of crocins can reach $22.76 \%$ which is higher than ultrasonic extraction method. Therefore, homogenate extraction is an effective way to extract crocins from saffron with higher extraction yield and shorter extraction time.

\section{Introduction}

Saffron, which is the red stigma of Crocus sativus L., is widely cultivated in Iran, India, Pakistan, Greece, Italy, China, Japan, Azerbaijan, and so on [1]. It is considered to be one of the world's most expensive spices. The main components in saffron are carotenoids like crocins and crocetin, monoterpene aldehydes like picrocrocin and safranal, and flavonoids. Crocin, which is responsible for the color of saffron, is the most abundant compound in saffron [2]. It has been known that saffron is useful to cure various illnesses, for example, neuronal diseases like depressive disorder [3]; it is also useful for preventing diabetes complication [4], metabolic syndrome [5], and cancer [6] in both clinical trials and animal model studies. It also has various biological activities extensively reviewed by Bathaie's [7] and Hosseinzadeh's research groups [8]. It is also safe and nontoxic in both human and animal studies $[7,9]$.
To our knowledge, extraction methods for crocins in addition to the chromatographic techniques [10] are including ultrasonic-assisted extraction [11-13], molecularly imprinted polymer solid-phase extraction [14], high-voltage pulsed electric field-assisted extraction [15], solid-liquid dynamic extraction [13], and supercritical fluid extraction [16]. These procedures give good extraction yields, but most of them require long time or special instruments to complete the process and some of them are not suitable for industrial production at present. Because of the instability of crocins in solution and in the light, the new extraction technology used in crocins extraction from saffron needs to shorten the extraction time and procedure. Meanwhile the homogenate extraction method can just meet the above requirements. Under the action of high-speed shear machine and cutting fluid, it can extract chemical compounds from material in solvent within a very short time without heating and pressure. Moreover, the process of homogenate extraction is carried 


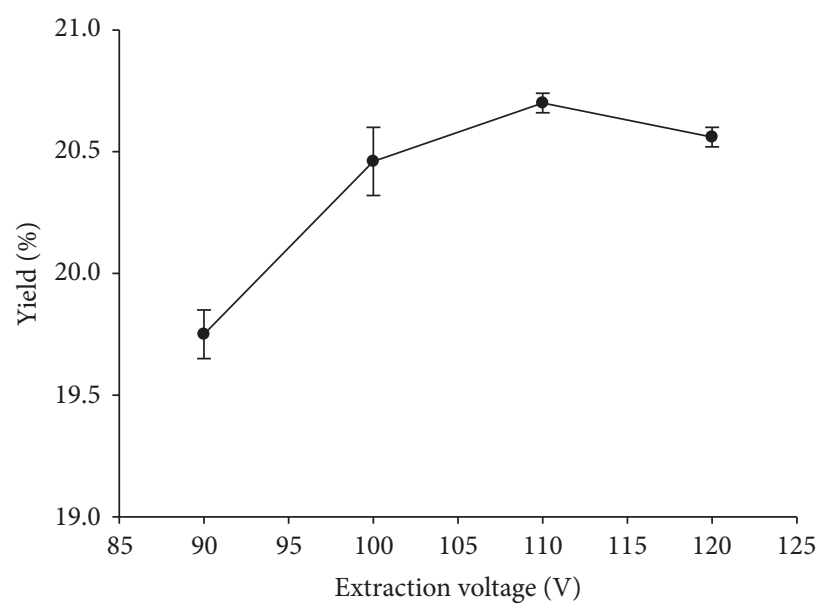

(a)

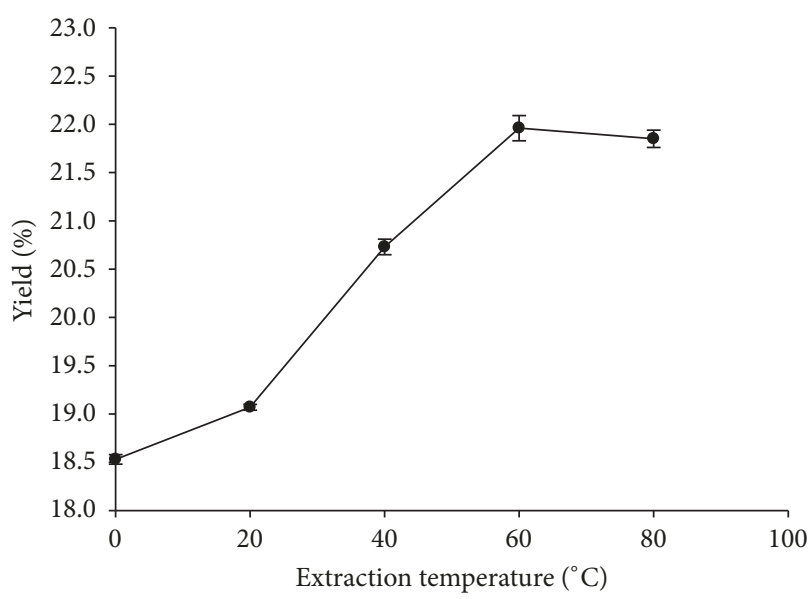

(c)

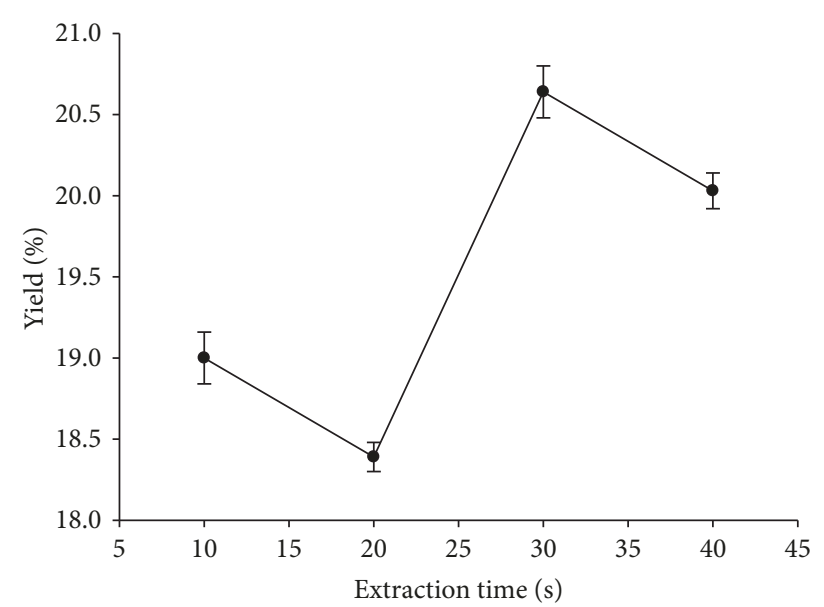

(b)

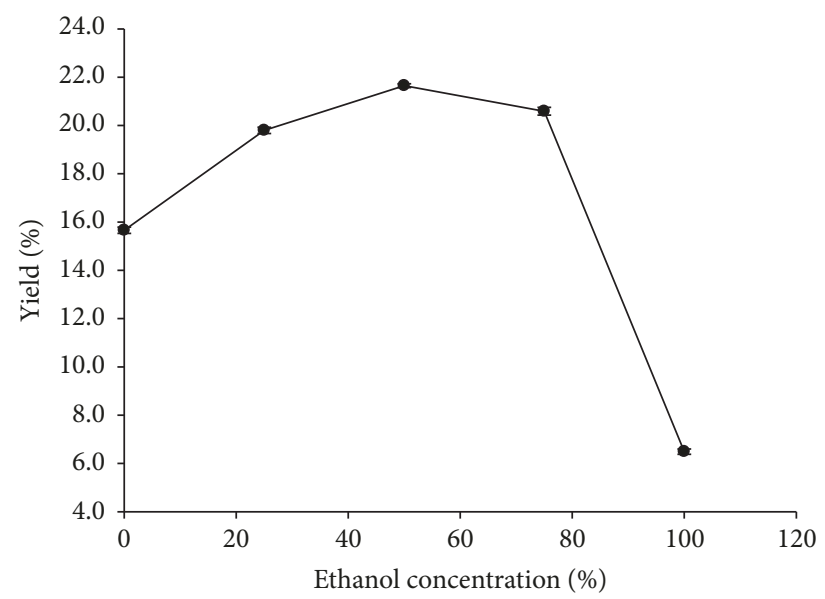

(d)

FIGURE 1: The effect of extraction voltage (a), extraction time (b), extraction temperature (c), and ethanol concentration (d) on the yields of crocins from saffron $(n=3)$.

out in stainless steel tanks; it can also effectively reduce the impact of light on crocins. It has been used to extract camptothecin and hydroxycamptothecin from Camptotheca acuminata leaves [17] and isoflavones from soybean meal [18], which has been proved to be an effective extraction method. However, as far as we know, there was no report about its application on crocins extraction from saffron. In this study, it was applied to extract crocins from saffron and optimized by response surface method for the first time.

\section{Results}

2.1. Linear Relationship of Calibration Curve. Linear Relationship of Calibration Curve Regression Equation of crocin1 was obtained by regression analysis of absorbance value of crocin-1 solution ( $y$-axis) against concentration ( $x$-axis, $\mu \mathrm{g} / \mathrm{ml})$. Linear equation $(Y=0.0665 X+0.0108)$ had excellent correlation coefficient, $R=0.9995(n=5)$.

\subsection{Singer Factor Experiment}

2.2.1. Effect of Extraction Voltage on Crocins Yield. As shown in Figure 1(a), the crocins yield increased with extraction voltage from 90 to $110 \mathrm{~V}$ and reached $20.70 \pm 0.04(\%)$ at $110 \mathrm{~V}$. After this point, it slightly reduced with voltage increasing. Therefore, 100-120 V was selected as the extraction voltage in the Box-Behnken design process.

2.2.2. Effect of Extraction Time on Crocins Yield. Figure 1(b) showed that the crocins yield reached $20.64 \pm 0.16(\%)$ at $30 \mathrm{~s}$. From 10 to $20 \mathrm{~s}$ and 30 to $40 \mathrm{~s}$, the crocins yield decreased with extraction time increasing while it increased with extraction time increasing from 20 to $30 \mathrm{~s}$. So in the Box-Behnken design process extraction time was set from 20 to $40 \mathrm{~s}$.

2.2.3. Effect of Extraction Temperature on Crocins Yield. As shown in Figure 1(c), the crocins yield increased slowly with extraction temperature from 0 to $60^{\circ} \mathrm{C}$ to reach $21.96 \pm 0.13$ (\%) at $60^{\circ} \mathrm{C}$; then it was relatively stable with slight decrease when temperature increased from 60 to $80^{\circ} \mathrm{C}$. Therefore, $40-80^{\circ} \mathrm{C}$ was selected as the extraction temperature in the Box-Behnken design test.

2.2.4. Effect of Ethanol Concentration on Crocins Yield. According to Figure $1(\mathrm{~d})$, the crocins yield increased with ethanol concentration from 0 to $50 \%$ and reached $21.65 \pm 0.08$ 
TABLE 1: Results of the variance analysis of regression model.

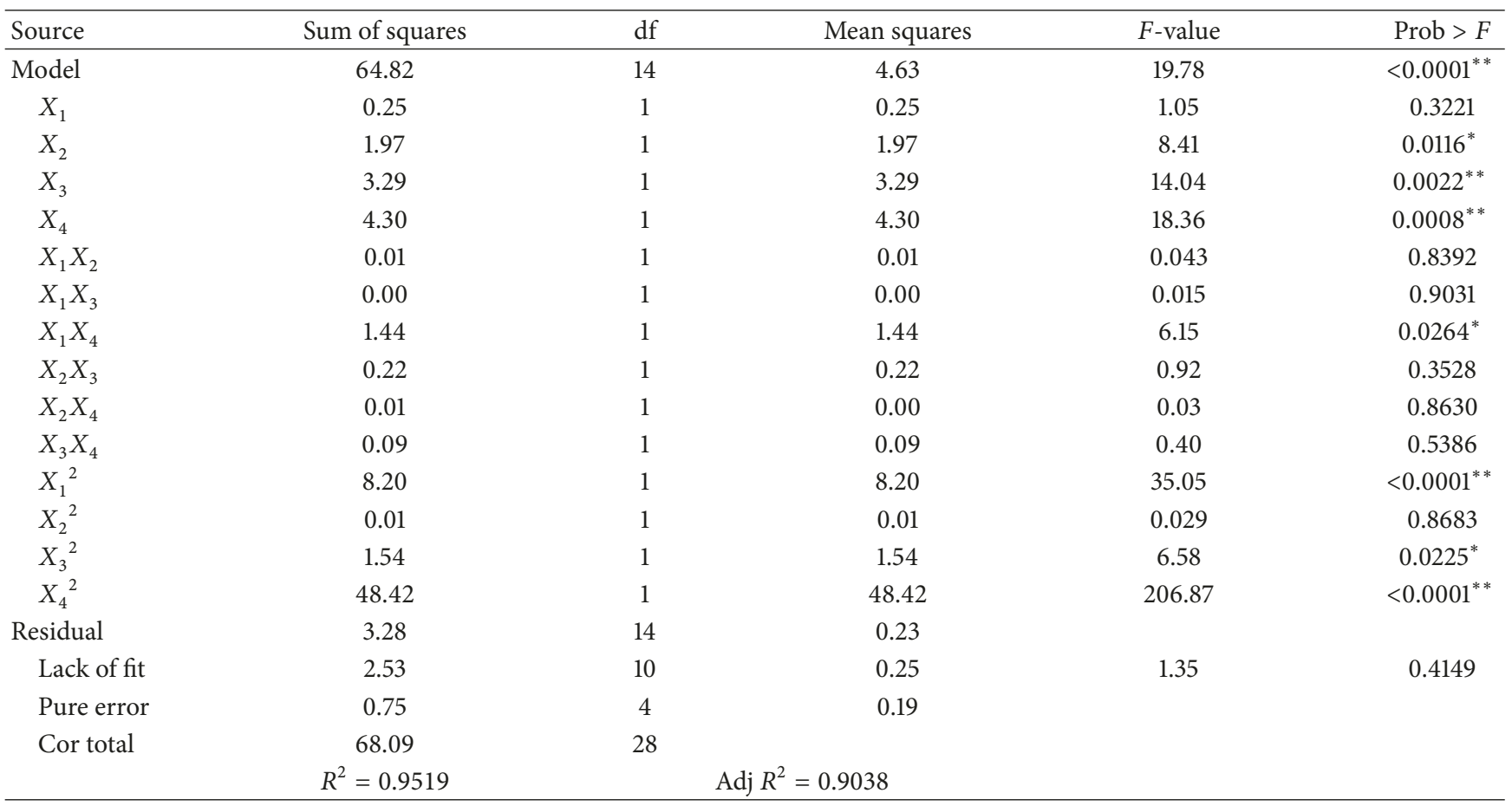

${ }^{*} p<0.05$ : significant; ${ }^{* *} p<0.01$ : highly significant; df: degree of freedom.

(\%) at $50 \%$. Then, it was also relatively stable with slight decrease when ethanol concentration increased to $75 \%$. But the crocins yield sharply decreased to $6.49 \pm 0.11$ (\%) when the ethanol concentration increased from 75 to $100 \%$. Thus, ethanol concentration was suitable to set at $25-75 \%$ in the Box-Behnken design process.

2.3. Response Surface Optimization of Homogenate Extraction Conditions. Design-Expert 8.0.6 Trial software was used to analyze the experimental data of response surface and the quadratic polynomial equation was presented in

$$
\begin{aligned}
Y= & 22.11+0.14 \times X_{1}+0.40 \times X_{2}+0.52 \times X_{3}-0.60 \\
& \times X_{4}+0.050 \times X_{1} \times X_{2}-0.030 \times X_{1} \times X_{3} \\
& -0.60 \times X_{1} \times X_{4}+0.23 \times X_{2} \times X_{3}-0.042 \times X_{2} \\
& \times X_{4}-0.15 \times X_{3} \times X_{4}-1.12 \times X_{1}^{2}-0.032 \\
& \times X_{2}^{2}-0.49 \times X_{3}^{2}-2.73 \times X_{4}^{2}
\end{aligned}
$$

According to the ANOVA results (Table 1), the $p$ values of $X_{2}, X_{3}, X_{4}, X_{1} X_{4}, X_{1}{ }^{2}$, and $X_{4}{ }^{2}$ were smaller than 0.05 , indicating they had a significant influence on the yield of crocins from saffron. The $R^{2}$ and Adj $R^{2}$ values of (1) were 0.9519 and 0.9038 , respectively, which meant the predicted values of (1) had great correlation with real experimental values between the chosen variables including extraction voltage, extraction time, ethanol concentration, and extraction temperature. The correlation between predicted values and real experimental values was showed in Figure 2. As a result of lack of fit test, the fitness of the model was good, because the $p$ value of lack of fit was 0.4149 , which was greater than 0.05 .

The $3 \mathrm{D}$ response surface plots were made by DesignExpert software in order to get a better visualization between the relations of extraction variables and crocins yields (Figure 3). Among the plots, the interaction of extraction voltage and extraction temperature had significant effects on crocins yield at a fixed time and ethanol concentration and the highest extract yield was achieved when the voltage and temperature were set nearly $110 \mathrm{~V}$ and $50^{\circ} \mathrm{C}$, respectively. From other plots, the yield variations of crocins can also be seen, but they were not statistically significant.

The optimal values of extraction voltage, extraction time, ethanol concentration, and extraction temperature were also calculated by Design-Expert software, which were $111.2 \mathrm{~V}$, $40 \mathrm{~s}, 70 \%$, and $50^{\circ} \mathrm{C}$, respectively. Under the above conditions, the maximum predicted yield of crocins was $22.84 \%$.

In order to compare the predicted value with the actual result, an experiment was performed for three times and the extraction parameters were set at $111 \mathrm{~V}, 40 \mathrm{~s}, 70 \%$, and $50^{\circ} \mathrm{C}$ for operational convenience. The mean value of these three experiments was $22.76 \%$, which was slightly lower than the one predicted by equation. This result demonstrated that the optimized model adequately reflected the actual extraction process of crocins.

2.4. Comparison of Different Extraction Methods. Homogenate extraction was compared with ultrasonic extraction for crocins from saffron. The ultrasonic extraction process was also optimized by RSM, and the optimal parameters of ultrasonic extraction were $41 \%, 40^{\circ} \mathrm{C}$, and $29 \mathrm{~min}$ for ethanol concentration, extraction temperature, and extraction time, 


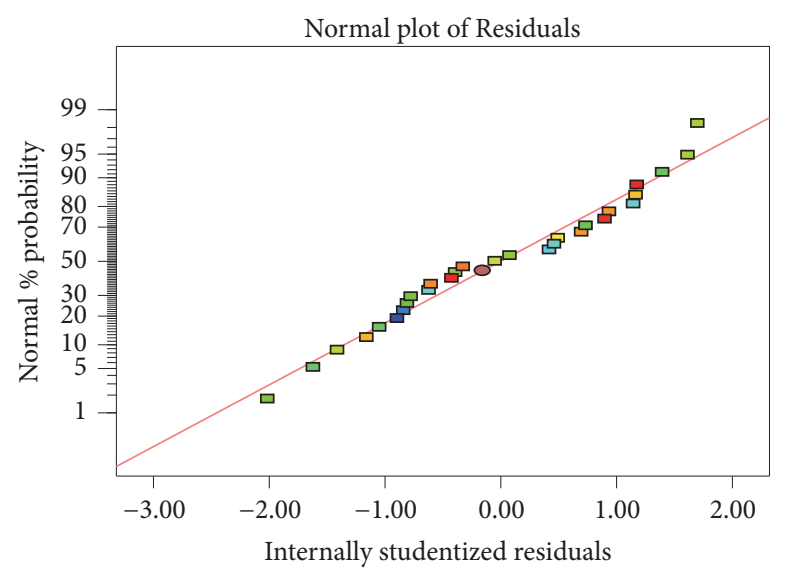

FIGURE 2: The correlation between predicted values obtained by the quadratic polynomial equations and actual experimental values.

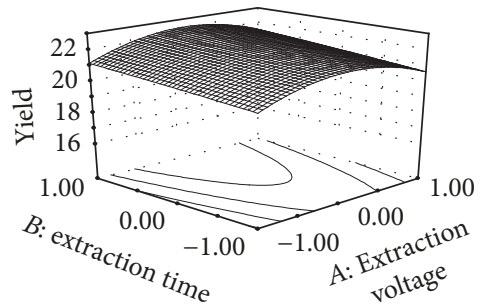

(a)

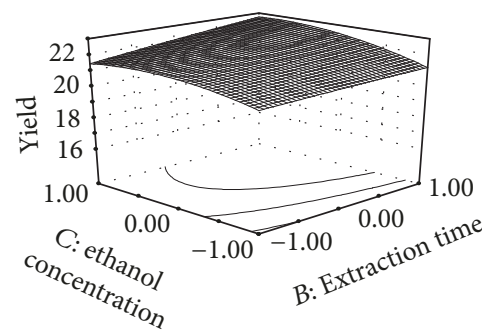

(d)

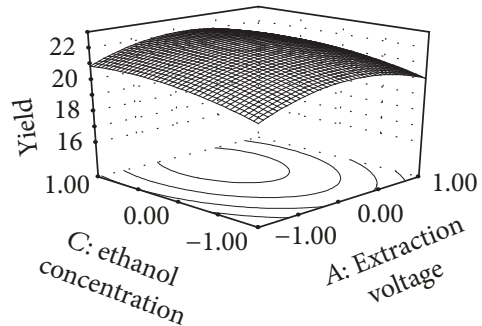

(b)

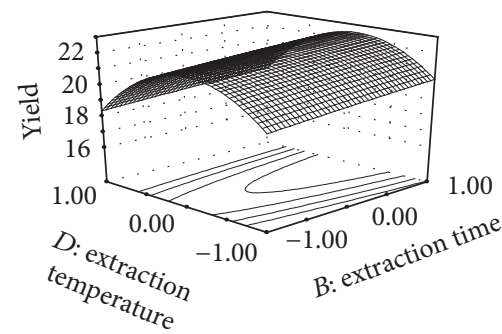

(e)

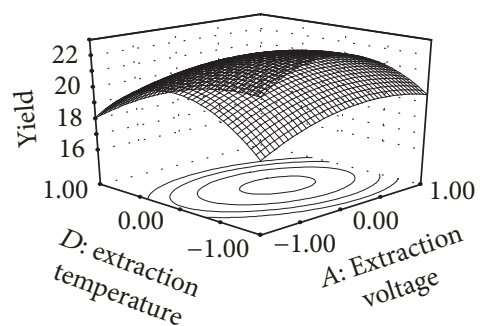

(c)

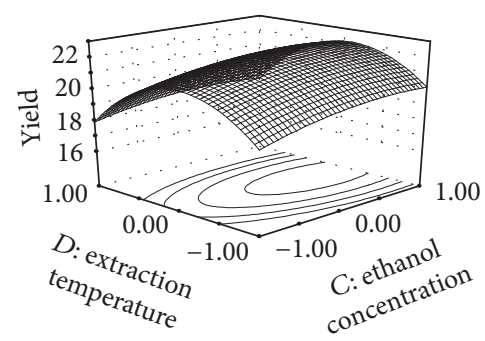

(f)

FIGURE 3: Response surface plot showing the effect of extraction voltage and extraction temperature on the yield of crocins from saffron.

respectively. Under this condition, the extraction yield of crocins for ultrasonic extraction was $18.51 \%$, which was $22.96 \%$ lower than that in homogenate extraction. In addition to the higher extraction yield of crocins, the extraction time of homogenate extraction was also significantly shorter than ultrasonic extraction. Hence, it was worth noticing that homogenate extraction was a good alternative to extract crocins from saffron.

\section{Materials and Methods}

3.1. Materials and Reagents. Saffron, which was authenticated by Professor Ping Wang, was purchased from Jiande Sandu farm, Hangzhou, Zhejiang Province of China. Crocin-1 was isolated from saffron in our laboratory. Other reagents were all of analytical grade.

3.2. Apparatus. Homogenate extraction was carried out on JHBE-50A homogenate extractor (Golden Star Technology,
TABLE 2: Coded levels of independent variables used in the RSM design.

\begin{tabular}{lccc}
\hline Factor & \multicolumn{3}{c}{ Level } \\
& -1 & 0 & 1 \\
\hline Extraction voltage $X_{1}(\mathrm{~V})$ & 100 & 110 & 120 \\
Extraction time $X_{2}(\mathrm{~s})$ & 20 & 30 & 40 \\
Ethanol concentration $X_{3}(\%)$ & 25 & 50 & 75 \\
Extraction temperature $X_{4}\left({ }^{\circ} \mathrm{C}\right)$ & 40 & 60 & 80 \\
\hline
\end{tabular}

Inc., Ltd., Zhengzhou, China). UV-Vis spectrophotometer (756 PC) was purchased from Shanghai Spectrum Instruments Co., Ltd. (Shanghai, China).

3.3. Total Crocins Determination. The total crocins content was determined using a standard curve with crocin-1. Firstly, 
TABLE 3: Experimental design and responses of the dependent variables to the extract parameters.

\begin{tabular}{lccccc}
\hline Numbers & $X 1$ & $X 2$ & Factors & $X 3$ & Yield/\% \\
\hline 1 & 0 & 1 & -1 & 0 & 20.80 \\
2 & -1 & -1 & 0 & 0 & 20.48 \\
3 & 1 & 0 & 0 & -1 & 19.09 \\
4 & 0 & 0 & 0 & 0 & 22.62 \\
5 & -1 & 0 & -1 & 0 & 19.55 \\
6 & 0 & 0 & -1 & -1 & 19.25 \\
7 & 1 & 0 & 0 & 21.77 \\
8 & 0 & 1 & 1 & -1 & 20.67 \\
9 & -1 & 0 & 0 & -1 & 18.24 \\
10 & 1 & 0 & 1 & 16.92 \\
11 & 0 & -1 & 0 & 20.88 \\
12 & -1 & 0 & 0 & 0 & 21.53 \\
13 & 0 & -1 & 0 & 1 & 18.53 \\
14 & 0 & 1 & 0 & 1 & 19.34 \\
15 & -1 & 1 & 0 & 20.28 \\
16 & 0 & 0 & 0 & 21.61 \\
17 & 1 & 0 & 0 & 20.52 \\
18 & 0 & 1 & 1 & 18.47 \\
19 & 0 & 0 & 1 & 18.47 \\
20 & -1 & 0 & 0 & 0 & 21.97 \\
21 & 0 & 0 & 0 & 22.50 \\
22 & 0 & 0 & 1 & 17.66 \\
23 & 0 & 0 & 1 & 21.77 \\
24 & 0 & 0 & 0 & 19.17 \\
25 & 0 & -1 & 0 & 20.68 \\
26 & 0 & 0 & 0.15 \\
27 & 0 & 0 & 0 & 21.85 \\
28 & 0 & 1 & 0 & 21.29 \\
29 & 0 & 0 & 0 & 22.62 \\
\hline
\end{tabular}

the stock solution of standard crocin- $1(1.03 \mathrm{mg} / \mathrm{ml})$ was prepared with ultrapure water, and a serial dilution of $2.57 \mu \mathrm{g} / \mathrm{ml}$ up to $10.27 \mu \mathrm{g} / \mathrm{ml}$ was prepared. Subsequently, all standard solutions were determined by UV-Vis spectroscopy within the $440 \mathrm{~nm}$. By plotting absorbance value of each standard solution ( $y$-axis) against concentration $(x$-axis, $\mathrm{mg} / \mathrm{ml})$, a regression equation was established. The crocins content in extraction solution was measured in triplicate and using the standard curve; after that, the extraction yield of crocins was obtained using

$$
\begin{aligned}
& \text { Extraction Yield (\%) } \\
& \begin{aligned}
= & \frac{\text { The mass of crocins in extraction solution }}{\text { The mass of sample }} \\
& \times 100 .
\end{aligned}
\end{aligned}
$$

3.4. Homogenate Extraction Process. Under the designed extraction voltage, extraction time, extraction temperature, and ethanol concentration, saffron powder $(0.1 \mathrm{~g})$ mixed with ethanol $(100 \mathrm{ml})$ was extracted by homogenate extractor; then the sample was centrifuged at $4700 \mathrm{rpm}$ for $10 \mathrm{~min}$. After that, the supernatant was filtered and collected to determine the content of crocins by UV-Vis spectrophotometer at $440 \mathrm{~nm}$. The whole extraction process was carried out in triplicate.

3.5. Experimental Design. According to the results of single factor experiments, response surface methodology based on Box-Behnken design (BBD) was applied to identify the best extraction conditions for crocins by using four variables, the extraction voltage $(X 1)$, extraction time $(X 2)$, ethanol concentration $(X 3)$, and extraction temperature $(X 4)$, respectively. And the average of crocins yield $(Y)$ was taken as the response of the design experiments. The above variables listed in Table 2 were coded according to the following equation:

$$
x i=\frac{X_{i}-X_{0}}{\Delta X},
$$

where $x i$ is the coded value of a variable, $X_{i}$ is the real value of a variable, $X_{0}$ is the actual value of a variable at the center point, and $\Delta X$ is the step change value.

As showed in Table 3, a total of 29 experimental runs for BBD were carried out. The independent variables and 
the crocins yield were correlated by the following quadratic polynomial model:

$$
Y=\gamma_{0}+\sum \gamma_{i} x_{i}+\sum \gamma_{i i} x_{i}^{2}+\sum \gamma_{i j} x_{i} x_{j}
$$

where $Y$ is the predicted response; $\gamma_{0}, \gamma_{i}, \gamma_{i i}$, and $\gamma_{i j}$ are the coefficients for constant, linear, squared, and interaction terms, respectively.

\section{Conclusions}

In this article, we use crocin- 1 as a standard substance to determine the content of crocins and decide which extraction process is better, because all the crocins such as trans-4-GG (crocin-1), trans-3-Gg (crocin-2), trans-2-gg, and crocetin have similar UV absorption wavelength. The test results of crocins in extraction solution were not interfered by safranal, picrocrocin, and flavonoids, because these compounds have no absorption at $440 \mathrm{~nm}$ except for crocins

In this study, an efficient homogenate extraction method was successfully applied to extract crocins from saffron. RSM was used to optimize the extraction conditions with high extraction yield. The optimum conditions were $111 \mathrm{~V}$, $40 \mathrm{~s}, 70 \%$, and $50^{\circ} \mathrm{C}$ for extraction voltage, extraction time, ethanol concentration, and extraction temperature, respectively. Under these conditions, extraction yield of crocins was much higher than ultrasonic extraction method, in a significantly shorter extraction time. All these data showed that homogenate extraction method was a more efficient procedure for extracting crocins from saffron.

\section{Conflicts of Interest}

The authors declare no conflicts of interest.

\section{Acknowledgments}

The authors are grateful to China Postdoctoral Science Foundation (no. 2016M592016) and Key Project of Science and Technology Department of Zhejiang Province (no. 2015C02032) for financial help.

\section{References}

[1] A. Ramadan, G. Soliman, SS. Mahmoud, SM. Nofal, and RF. Abdel-Rahman, "Evaluation of the safety and antioxidant activities of Crocus sativus, and Propolis, ethanolic extracts," Journal of Saudi Chemical Society, vol. 16, pp. 13-21, 2012.

[2] M. Kabiri, H. Rezadoost, and A. Ghassempour, "A comparative quality study of saffron constituents through HPLC and HPTLC methods followed by isolation of crocins and picrocrocin," LWT- Food Science and Technology, vol. 84, pp. 1-9, 2017.

[3] A. Talaei, M. Hassanpour Moghadam, S. A. Sajadi Tabassi, and S. A. Mohajeri, "Crocin, the main active saffron constituent, as an adjunctive treatment in major depressive disorder: A randomized, double-blind, placebo-controlled, pilot clinical trial," Journal of Affective Disorders, vol. 174, pp. 51-56, 2015.

[4] E. Altinoz, Z. Oner, H. Elbe, Y. Cigremis, and Y. Turkoz, "Protective effects of saffron (its active constituent, crocin) on nephropathy in streptozotocin-induced diabetic rats," Human \& Experimental Toxicology, vol. 34, no. 2, pp. 127-134, 2015.
[5] I. Nikbakht-Jam, M. Khademi, M. Nosrati et al., "Effect of crocin extracted from saffron on pro-oxidant-anti-oxidant balance in subjects with metabolic syndrome: A randomized, placebo-controlled clinical trial," European Journal of Integrative Medicine, vol. 8, no. 3, pp. 307-312, 2016.

[6] J. Escribano, G.-L. Alonso, M. Coca-Prados, and J.-A. Fernández, "Crocin, safranal and picrocrocin from saffron (Crocus sativus L.) inhibit the growth of human cancer cells in vitro," Cancer Letters, vol. 100, no. 1-2, pp. 23-30, 1996.

[7] S. Z. Bathaie, S. Mousavi, and Z., "New Applications of saffron and molecular mechanism of its constituents action," Critical Reviews in Food Science and Nutrition, vol. 50, pp. 761-786, 2010.

[8] S. H. Alavizadeh and H. Hosseinzadeh, "Bioactivity assessment and toxicity of crocin: a comprehensive review, Food and Chemical Toxicology, vol. 64, pp. 65-80, 2014.

[9] M.-H. Modaghegh, M. Shahabian, H.-A. Esmaeili, O. Rajbai, and H. Hosseinzadeh, "Safety evaluation of saffron (Crocus sativus) tablets in healthy volunteers," Phytomedicine, vol. 15, no. 12, pp. 1032-1037, 2008.

[10] H. Zhang, Y. Zeng, F. Yan et al., "Semi-preparative isolation of crocins from saffron (Crocus sativus L.)," Chromatographia, vol. 59, no. 11-12, pp. 691-696, 2004.

[11] R. Kadkhodaee and A. Hemmati-Kakhki, "Ultrasonic extraction of active compounds from saffron," Acta Horticulturae, vol. 739, pp. 417-425, 2007.

[12] A. Kyriakoudi, A. Chrysanthou, F. Mantzouridou, and M. Z. Tsimidou, "Revisiting extraction of bioactive apocarotenoids from Crocus sativus L. dry stigmas (saffron)," Analytica Chimica Acta, vol. 755, pp. 77-85, 2012.

[13] L. Ferrara, D. Naviglio, and M. Gallo, "Extraction of Bioactive Compounds of Saffron (Crocus sativus L.) by Ultrasound Assisted Extraction (UAE) and by Rapid Solid-Liquid Dynamic Extraction (RSLDE)," European Scientific Journal, vol. 10, pp. 113, 2014.

[14] S. A. Mohajeri, H. Hosseinzadeh, F. Keyhanfar, and J. Aghamohammadian, "Extraction of crocin from saffron (Crocus sativus) using molecularly imprinted polymer solid-phase extraction," Journal of Separation Science, vol. 33, no. 15, pp. 2302-2309, 2010.

[15] A. Pourzaki, H. Mirzaee, and A. Hemmati Kakhki, "Using pulsed electric field for improvement of components extraction of saffron (Crocus sativus) stigma and its pomace," Journal of Food Processing and Preservation, vol. 37, no. 5, pp. 1008-1013, 2013.

[16] M. G. Goleroudbary and S. M. Ghoreishi, "Response surface optimization of Safranal and Crocin extraction from Crocus sativus L. via supercritical fluid technology," The Journal of Supercritical Fluids, vol. 108, pp. 136-144, 2016.

[17] W.-G. Shi, Y.-G. Zu, C.-J. Zhao, and L. Yang, "Homogenate extraction technology of camptothecine and hydroxycamptothecin from camptotheca acuminata leaves," Journal of Forestry Research, vol. 20, no. 2, pp. 168-170, 2009.

[18] X. Y. Zhu, H. M. Lin, J. Xie, S. S. Chen, and P. Wang, "Homogenate extraction of isoflavones from soybean meal by orthogonal design," Journal of Scientific and Industrial Research, vol. 70, pp. 455-460, 2011. 

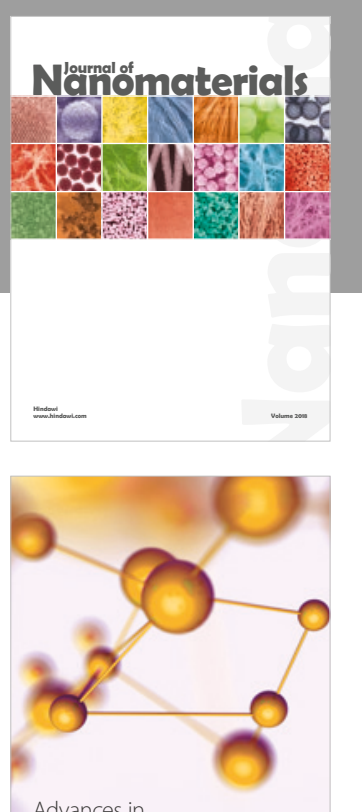

Physical Chemistry
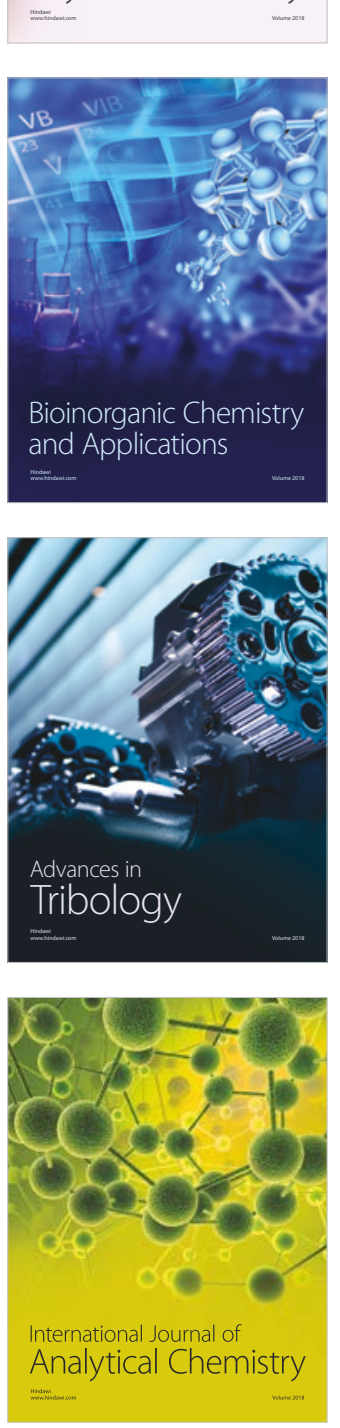

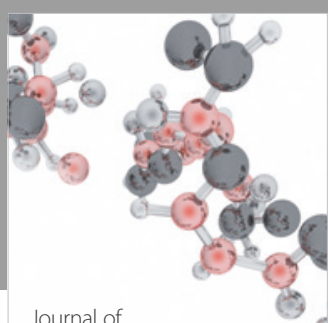

Analytical Methods

in Chemistry

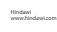

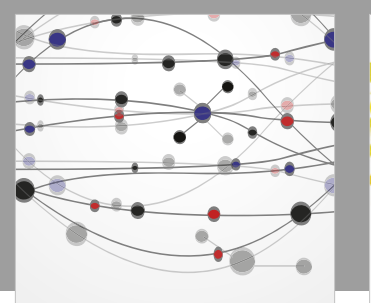

The Scientific World Journal

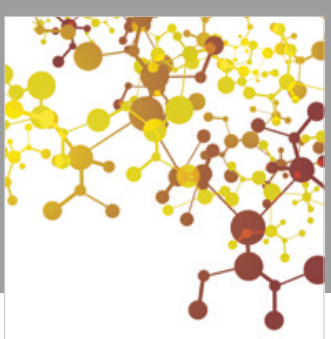

Journal of

Applied Chemistry
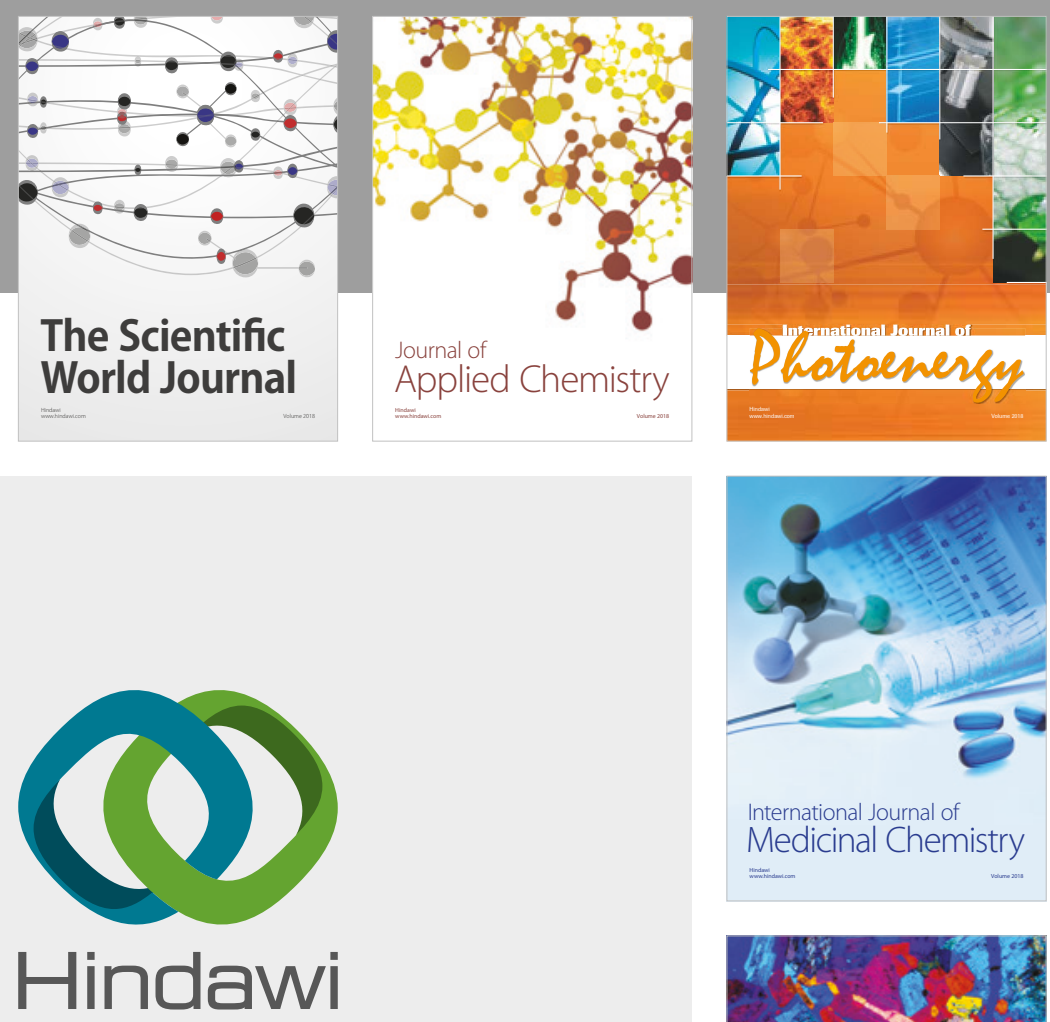

Submit your manuscripts at

www.hindawi.com
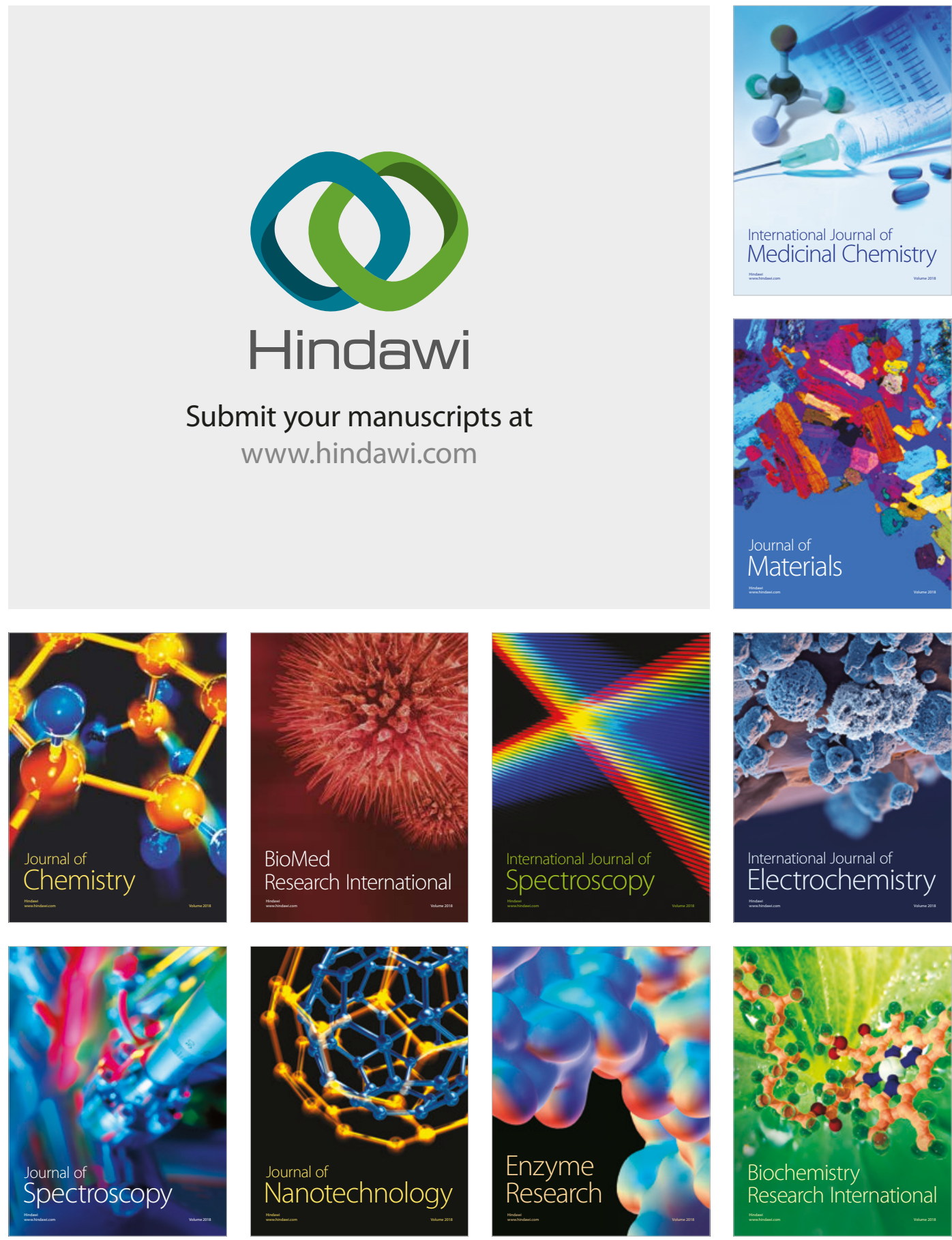
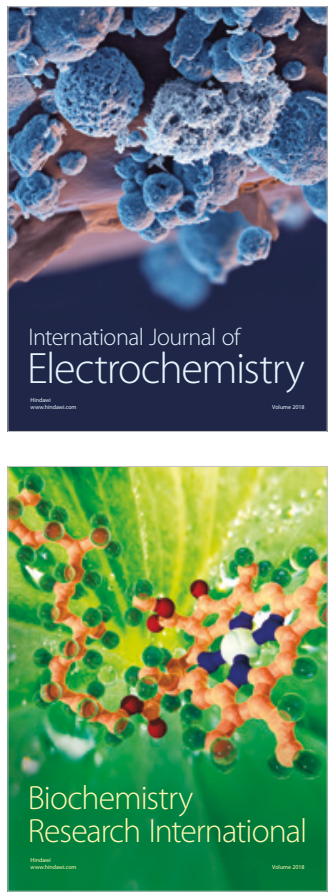